\title{
Progress in ARDS research: a protection racket?
}

\author{
J Dakin, T W Evans
}

The acute respiratory distress syndrome in adults (ARDS) may complicate a wide variety of serious medical and surgical conditions, not all of which involve the lung directly. ${ }^{1}$ The pathogenesis remains unclear, but involves neutrophil recruitment to the alveoli and inflammatory pathway activation leading to increased permeability of the alveolarcapillary membrane and disordered vascular control, manifest clinically as pulmonary oedema formation and refractory hypoxaemia. Compliance is reduced and work of breathing is increased to the extent that most patients require endotracheal intubation and mechanical ventilatory support. ${ }^{2}$ Despite the recent publication of withincentre studies showing a fall in mortality, ${ }^{34}$ some $40-60 \%$ of patients with ARDS fail to survive with most of the deaths being attributable to multiple organ system failure.

It has been appreciated for some years that mechanical ventilation can itself exacerbate pre-existing alveolar injury. This may develop directly as a result of barotrauma and so called "volutrauma", or indirectly through the adverse effects of increasing the inspired oxygen concentration to obviate hypoxaemia. Thus, studies in experimental animals have suggested that injury rises markedly above an end inspiratory (or plateau) pressure of $35 \mathrm{~cm} \mathrm{H}_{2} \mathrm{O}$. Secondly, computed tomographic scanning in patients with ARDS subjected to varying levels of positive end expiratory pressure (PEEP) has shown that dependent consolidated areas of lung tend to remain poorly compliant and underventilated, and the application of conventional tidal volumes of the order of $10 \mathrm{ml} / \mathrm{kg}$ can overdistend the spared functioning regions. ${ }^{6}$ Consequently, the last decade has seen the emergence of ventilation strategies characterised by lower tidal and minute volumes. The cost of such an approach is reduced alveolar ventilation, which may further compromise oxygenation and $\mathrm{CO}_{2}$ elimination. To accommodate this, target arterial haemoglobin saturation has been reduced to $90 \%$, or slightly less, without compromising oxygen delivery. Higher respiratory rates of up to 35 breaths/min may be needed. Reduced $\mathrm{CO}_{2}$ elimination necessitates tolerating respiratory acidosis or correction with bicarbonate. Finally, the application of levels of PEEP above the lower inflection point of the pulmonary pressure-volume curve increases functional residual capacity with a view to keeping more alveoli open throughout the respiratory cycle, thereby reducing shear (the so called "open lung strategy").

The precise mode of ventilation applied to achieve these physiological end points has varied and studies have provided conflicting evidence concerning efficacy. Thus, an early randomised trial of tidal volumes of $<6 \mathrm{ml} / \mathrm{kg}$, a driving pressure above PEEP (titrated to the lower inflection point) of $20 \mathrm{~cm} \mathrm{H} \mathrm{H}_{2} \mathrm{O}$, and permissive hypercapnia compared with conventional support in patients with early ARDS showed a significant survival advantage (38\% mortality) although mortality in the control group (70\%) was also high. ${ }^{7}$ By contrast, subsequent studies limiting end inspiratory pressures to $25 \mathrm{~cm} \mathrm{H}_{2} \mathrm{O}$ and tidal volumes to $<10 \mathrm{ml} / \mathrm{kg}$ showed no mortality benefit, ${ }^{8}$ and patients at high risk of ARDS subjected to pressure $\left(30 \mathrm{~cm} \mathrm{H}_{2} \mathrm{O}\right)$ and volume limited ventilation $(8 \mathrm{ml} / \mathrm{kg})$ showed no increase in survival compared with conventionally supported controls. ${ }^{9}$ However, the results of a recently published, large scale trial performed in 861 patients by the US acute respiratory distress syndrome network (ARDSNET) have been more encouraging. ${ }^{10}$ Within 36 hours of the onset of refractory hypoxaemia sufficiently severe to meet the AmericanEuropean consensus guidelines criteria for acute lung injury (ALI, $\mathrm{PaO}_{2}: \mathrm{FiO}_{2}<300 \mathrm{~mm} \mathrm{Hg}$ ) in the presence of bilateral pulmonary infiltrates on chest radiography, subjects were randomised to receive conventional $(10 \mathrm{ml} / \mathrm{kg})$ or low volume ventilatory support ( $5 \mathrm{ml} / \mathrm{kg}) ; 75 \%$ of those in each group had developed ALI/ARDS in association with sepsis, pneumonia, or aspiration. The trial was stopped after interim analysis revealed mortality of $39.8 \%$ in the conventionally supported group and $31 \%$ in the lower tidal volume group, a reduction of $22 \%$. There were subtle, but probably significant, differences between the experimental protocol employed by the ARDSNET trialists and those of previous negative studies. Firstly, with respect to both tidal volume per actual body weight and plateau pressure, the two arms of this trial were further separated than any other. Although a volume cycled approach was employed, ventilation was frequently adjusted to maintain pressure levels, permitting wider tidal volume constraints. The mean plateau pressures recorded for the high and low volume arms, respectively, were therefore 33 and $25 \mathrm{~cm} \mathrm{H}_{2} \mathrm{O}$ compared with pressure differences of the order of 4.5-6.0 $\mathrm{cm} \mathrm{H}_{2} \mathrm{O}$ in the trials with negative results. Secondly, to attain the low plateau pressure in the protective ventilatory group tidal volumes of $5 \mathrm{ml} / \mathrm{kg}$ were used, which is lower than those used in previous trials. (Although the protocol stipulated $6 \mathrm{ml} / \mathrm{kg}$, this was per $\mathrm{kg}$ of weight predicted from measured height. The actual weight of their population exceeded this by an average of $20 \%$, reducing the actual tidal volume applied to nearer $5 \mathrm{ml} / \mathrm{kg}$.) Thirdly, in the ARDSNET study bicarbonate was used to correct the acidosis resulting from $\mathrm{CO}_{2}$ retention, a contentious innovation given recent evidence suggesting that buffering hypercapnic acidosis may worsen ALI. ${ }^{11}$

Not surprisingly, these results have been hailed as a significant advance in that they are the first to demonstrate mortality benefit of any intervention applied to patients with severe lung injury in a randomised controlled fashion. Is such optimism justified, especially as most of the patients with ARDS who fail to survive succumb to multiorgan system failure? In fact, the current results complement a growing body of experimental evidence which suggest that high volume, high pressure ventilation may propagate alveolar inflammation. ${ }^{12}$ Furthermore, in the past year a landmark study in patients with ARDS revealed that high tidal volume ventilatory support can not only increase alveolar inflammation, but also disseminate inflammatory cytokines into the systemic circulation. ${ }^{13}$ What, then, are the implications of this study for those clinicians involved in the care of patients with severe ALI? Two conclusions seem irrefutable. Firstly, ventilator strategy does seem to influence outcome and protective strategies aimed principally at reducing tidal volume in order to restrict plateau pressures should be introduced. Secondly, the results highlight the importance of the injured lung as the potential source of a systemic inflammatory response with potentially fatal consequences. In this sense inappropriate ventilation can clearly impact adversely on mortality.

By contrast, a number of important questions remain unanswered. Firstly, is a single approach to ventilation appropriate for all patients with acute severe lung injury? Recent studies suggest that patients with direct alveolar 
injury leading to ARDS may have differing physiological characteristics and ventilatory responses from those suffering indirect pulmonary insults. ${ }^{14}{ }^{15}$ The ARDSNET study made no attempt to stratify patients in this fashion and enrolled patients with ALI as well as ARDS. Moreover, patients were enrolled within 36 hours of the onset of lung injury, although ARDS is a condition passing through exudative, inflammatory, and fibroproliferative histopathological phases over some 2-3 weeks; optimal ventilatory support may therefore change as the condition evolves. Secondly, aiming consistently for a tidal volume of $5 \mathrm{ml} / \mathrm{kg}$ may be inappropriate, especially if it necessitates bicarbonate infusion. Thirdly, these results suggest that a re-evaluation of extreme approaches to lung protection incorporated in high frequency oscillation and extracorporeal supportive techniques may be appropriate. ${ }^{16}$ Noninvasive (face mask) positive pressure ventilation might have similarly beneficial effects and has already been used successfully in these circumstances. ${ }^{17}$

In summary, the ARDSNET investigation in a sense confirms what we have suspected for some years - that lung protection is the name of the game. How far and how best this approach should be applied to improve mortality further in patients with ARDS still remains to be seen.

This work was supported in part by the British Lung Foundation.

Correspondence to: Professor T W Evans

t.evans@rbh.nthames.nhs.uk

J DAKIN

Unit of Critical Care, T W EVANS

Imperial College School of Medicine,

Royal Brompton Hospital,

London SW3 6NP, UK
1 Bernard GR, Artigas A, Brigham KL, et al. The American-European Consensus Conference on ARDS. Definitions, mechanisms, relevant outcomes, and clinical trial coordination. Am F Respir Crit Care Med 1994;149:818-24.

2 Wyncoll DLA, Evans TW. Acute respiratory distress syndrome. Lancet 1999;354:497-502.

3 Milberg JA, Davis DR, Steinberg KP, et al. Improved survival of patients with acute respiratory distress syndrome 1983-1993. FAMA 1995;273: 306-9.

4 Abel SJ, Finney SJ, Brett SJ, et al. Reduced mortality in association with the acute respiratory distress syndrome. Thorax 1998;53:292-4.

5 Tsuno K, Miura K, Takeya M, et al. Histopathologic pulmonary changes from mechanical ventilation at high peak airway pressures. Am Rev Respir Dis 1991;143:1115-20.

6 Pelosi P, Crotti S, Brazzi L, et al. Computed tomography in adult respiratory distress syndrome: what has it taught us? Eur Respir f 1996;9:1055-62.

7 Amato MB, Barbas CS, Medeiros DM, et al. Effect of a protectiveventilation strategy on mortality in the acute respiratory distress syndrome. ventilation strategy on mortality

8 Brochard L, Roudot-Thoraval F, Roupie E, et al. Tidal volume reduction for prevention of ventilator-induced lung injury in acute respiratory distress syndrome. Am f Respir Crit Care Med 1998;158:1831-8.

9 Stewart TE, Meade MO, Cook DJ, et al. Evaluation of a ventilation strategy to prevent barotrauma in patients at high risk for acute respiratory distress syndrome. N Engl f Med 1998;338:355-61.

10 The Acute Respiratory Distress Syndrome Network. Ventilation with lower tidal volumes as compared with traditional tidal volumes for acute lung injury and the acute respiratory distress syndrome. N Engl f Med 2000; 342:1301-8

11 Laffey JG, Engelberts D, Kavanagh BP. Buffering hypercapnic acidosis worsens acute lung injury. Am F Respir Crit Care Med 2000;161:141-6.

12 Tremblay LN, Slutsky AS. Ventilator-induced injury: from barotrauma to biotrauma. Proc Ass Am Phys 1998;110:482-8.

13 Ranieri VM, Suter PM, Tortorella C. Effect of mechanical ventilation on inflammatory mediators in patients with acute respiratory distress syndrome: a randomized controlled trial. ҰAMA 1999;282:54-61.

14 Gattinoni L, Pelosi P, Suter PM. Acute respiratory distress syndrome caused by pulmonary and extrapulmonary disease. Different syndromes? Am $\mathcal{F}$ Respir Crit Care Med 1998;158:3-11.

15 Suntharalingham G, Regan K, Keogh BF, et al. Influence of direct and indirect etiology on acute outcome and six month functional recovery in acute respiratory distress syndrome. Crit Care Med 2000 (in press).

16 Keogh BF, Evans TW. Extracorporeal membrane oxygenation: a breath of fresh air or yesterday's therapy? Thorax 1991;46:692-4.

17 Antonelli M, Conti G, Rocco M. A comparison of noninvasive positive-pressure ventilation and conventional mechanical ventilation in patients with acute respiratory failure. N Engl F Med 1998;339:429-35. 